\title{
Flow pattern and pressure drop for oil-water flows in and around $180^{\circ}$ bends
}

\author{
Paul Onubi Ayegba ${ }^{1,2} \cdot$ Lawrence C. Edomwonyi-Otu $^{2,3} \cdot$ Abdulkareem Abubakar $^{2} \cdot$ Nurudeen Yusuf $^{4}$
}

Received: 11 September 2020 / Accepted: 21 December 2020 / Published online: 6 January 2021

(C) The Author(s) 2021 OPEN

\begin{abstract}
Pressure drop and flow pattern of oil-water flows were investigated in a 19-mm ID clear polyvinyl chloride pipe consisting of $U$-bend with radius of curvature of $100 \mathrm{~mm}$. The range for oil and water superficial velocities tested was $0.04 \leq U_{\text {so }} \leq 0.950 \mathrm{~m} / \mathrm{s}$ and $0.13 \leq U_{s w} \leq 1.10 \mathrm{~m} / \mathrm{s}$, respectively. Measurements were carried out under different flow conditions in a test section that consisted of four different parts: upstream of the bend, at the bend and at two redeveloping flow locations after the bend. The result indicated that the bend had limited influence on downstream flow patterns. However, the shear forces imposed by the bend caused some shift flow pattern transition and bubble characteristics in the redeveloping flow section after the bend relative to develop flow before the bend. Generally, pressure gradient at all the test sections increased with both oil fraction and water superficial velocity and there was a sharp change of pressure gradient profile during phase inversion. The transition point where phase inversion occurred was always within the range of $0.4 \leq U_{\mathrm{sw}} \leq 0.54 \mathrm{~m} / \mathrm{s}$. Pressure losses differed at the various test sections, and the difference was strongly linked to the superficial velocity of the phases and the flow pattern. At high mixture velocity, pressure losses at the redeveloping section after the bend were higher than that at the bend and that for fully developed flows. At low mixture velocity, pressure losses at the bend are higher than in the straight sections. Pressure drop generally decreased with level of flow development downstream of the bend.
\end{abstract}

Keywords U-bend · Oil-water flow · Flow pattern · Pressure losses · Flow redevelopment

\section{List of symbols}

DP Pressure drop

$\mathrm{DP}_{\mathrm{L}} \quad$ Pressure gradient

$R \quad$ Radius of curvature

$d \quad$ Pipe diameter

$f \quad$ Friction factor

ST Stratified

DC Dual continuous

Do/w/w Dispersed oil in water and water

Do/w Dispersed oil in water

Dw/o Dispersed water in oil

\section{Subscripts \\ SO Superficial oil \\ SW Superficial water \\ M Mixture}

\section{Introduction}

Co-current flow of oil and water in pipeline is common in chemical, process and petroleum industry operations [1]. Data on flow development/redevelopment, pressure drop, flow pattern, phase mixing and interfacial characteristics are essential for optimal design and operation

\footnotetext{
$\triangle$ Lawrence C. Edomwonyi-Otu, uceclce@ucl.ac.uk| ${ }^{1}$ Department of Mechanical Engineering, University of California Berkeley, Berkeley, CA 94720, USA. ${ }^{2}$ Department of Chemical Engineering, Ahmadu Bello University, Zaria 810212, Nigeria. ${ }^{3}$ Department of Chemical and Petroleum Engineering, Delta State University, Abraka 330106, Nigeria. ${ }^{4}$ Department of Chemical and Petroleum Engineering, Bayero University, Kano 700231, Nigeria.
} 
in the industries as well as for modelling [2-4]. In petroleum exploration and transport, connate water or injected water from enhanced oil recovery operations flows along with oil. Also, in the transport of high viscosity oils, water injection into the annulus of pipelines has been used as a means of reducing friction losses and by extension reducing pumping power requirements [5-11].

Few investigations have been conducted on the co-current flow of oil and water in U-bends with the view of characterising the flow patterns and pressure gradient associated with such flows [12-14]. With regard to the effect of $U$-bends on post-bend flow pattern characteristics, it has been reported that it is strongly influenced by fluid properties such as oil viscosity. Sharma et al. [14] observed flow patterns in $U$-bend as well at $120 \times I D$ (hydrodynamically developed flow) before and after a $U$-bend for the flow of low viscosity oil (kerosene)-water in horizontal, upward and downward flow directions. The viscosity and density of kerosene used were $\mu_{\mathrm{o}}=1.2 \mathrm{~m} \mathrm{~Pa}$. s and $\rho_{\mathrm{o}}=787 \mathrm{~kg} / \mathrm{m}^{3}$, respectively, and the bend curvature ratio $(2 R / d)$ was 16.67. Besides film inversion (consequent upon centrifugal forces in the bend), flow patterns before and after the bend were largely similar in all three flow directions. This is not surprising considering that flow pattern observations were done at locations before and after the bend where flows are fully developed. In a separate investigation (with same experimental set-up) using high viscosity oil (lube), Sharma et al. [13] reported that the bend as well the direction of flow had significant influence on the downstream flow patterns. The viscosity and density of lube used are $\mu_{\mathrm{o}}=220 \mathrm{~m} \mathrm{~Pa}$. s and $\rho_{\mathrm{o}}=960 \mathrm{~kg} / \mathrm{m}^{3}$, respectively. Not only was the flow pattern before and after the bend different for a given flow direction, but it varied from one flow direction to the other. This may be associated with the significant difference in viscosity of kerosene and lube oil and the resulting difference in interfacial stress between kerosene-water and lube-water. Also, flow development is a function of Reynolds number which in turn depends on fluid viscosity. It should also be stated that they reported a predominance of core annular flow in their lube-water experiments and an additional flow pattern classified as droplet flow which was absent in the kerosene-water experiments of Sharma et al. [14]. The predominance of core annular flow is signature characteristics of high-viscosity oil-water flows, and similar behaviour has been reported for straight pipe flows [15]. Pietrzak [12] in their study of oil-water flow in horizontal U-bends categorised flow patterns in terms of the dominant continuous phase (oil dominant, W/O or water dominant, $\mathrm{O} / \mathrm{W})$. Their flow pattern characterisation was, however, limited to the $U$-bend and did not extend to effects of the bend on downstream flow patterns. Reports on oil-water phase distribution in the region of high flow disturbances immediately downstream of the bend are lacking in the open literature. In the current work, high-speed imaging technique was used to capture phase distribution immediately after the bend as well as at the bend and before the bend.

A few experimental and modelling studies have been done to study the pressure gradient of oil-water twophase flows in $U$-bends $[13,14,16]$. Analogous to straight pipe flows, pressure drop for oil-water two-phase flows in $U$-bends increases with flowrate of either of the phases and more so with the more viscous phase $[13,14,16]$. In the oil-water experiments of Sharma et al. $[13,14]$, they reported higher pressure drop in the bend relative to upstream and downstream straight pipe sections where flows were hydrodynamically developed. This is expected, considering the additional bend loss due to the action of centrifugal forces on the flow at the bend along with the fact that pressure measurements before and after the bend were done for hydrodynamically developed flows. Research on the characteristics (pressure drop and flow pattern) of redeveloping flows after the bend is lacking.

The behaviour of the fluid flow in bends is complex due to the action of centrifugal forces on the mixture along with the underdeveloped flow profile in bends [17-19]. Wall and interfacial frictions in oil-water two flows contribute the most to pressure gradient in developed flows in straight horizontal smooth pipes [20-23]. In curved pipes and for undeveloped flows, additional losses result from secondary flows and form drag consequent upon the effect of centrifugal forces and flow redistribution. The contribution of secondary flows to pressure gradient, relative to wall and interface shear stresses, is significant at moderate flowrates in turbulent flow regime. In single phase flow, when secondary flow contribution is significant, the pressure gradient in bends is markedly higher than that in straight pipes of comparable length $[18,19]$. The difference generally increases with curvature ratio and decreases with the length of the bend. At higher flowrates, the effect of turbulence and interfacial tension dominates. Although the pressure gradient of oil-water flows in bend and straight pipes (developed flow scenario) is expected to converge in the inertia dominant regime, there are limited data from which a definite conclusion may be drawn. In the current work, experimental measurement of pressure gradient for oil-water flows at flowrates was carried out with the view of characterising the pressure gradient of such flows. Furthermore, pressure gradient for redeveloping oil-water flows after bends results from the combination of wall friction, interfacial friction and phase redistribution (form drag). While pressure drop due to wall and interfacial friction is primarily functions of fluid rheological properties and flowrate, the contribution of flow redistribution is a strong function, the level of flow disturbance 
Fig. 1 Sketches of flow patterns in and around $U$-bend
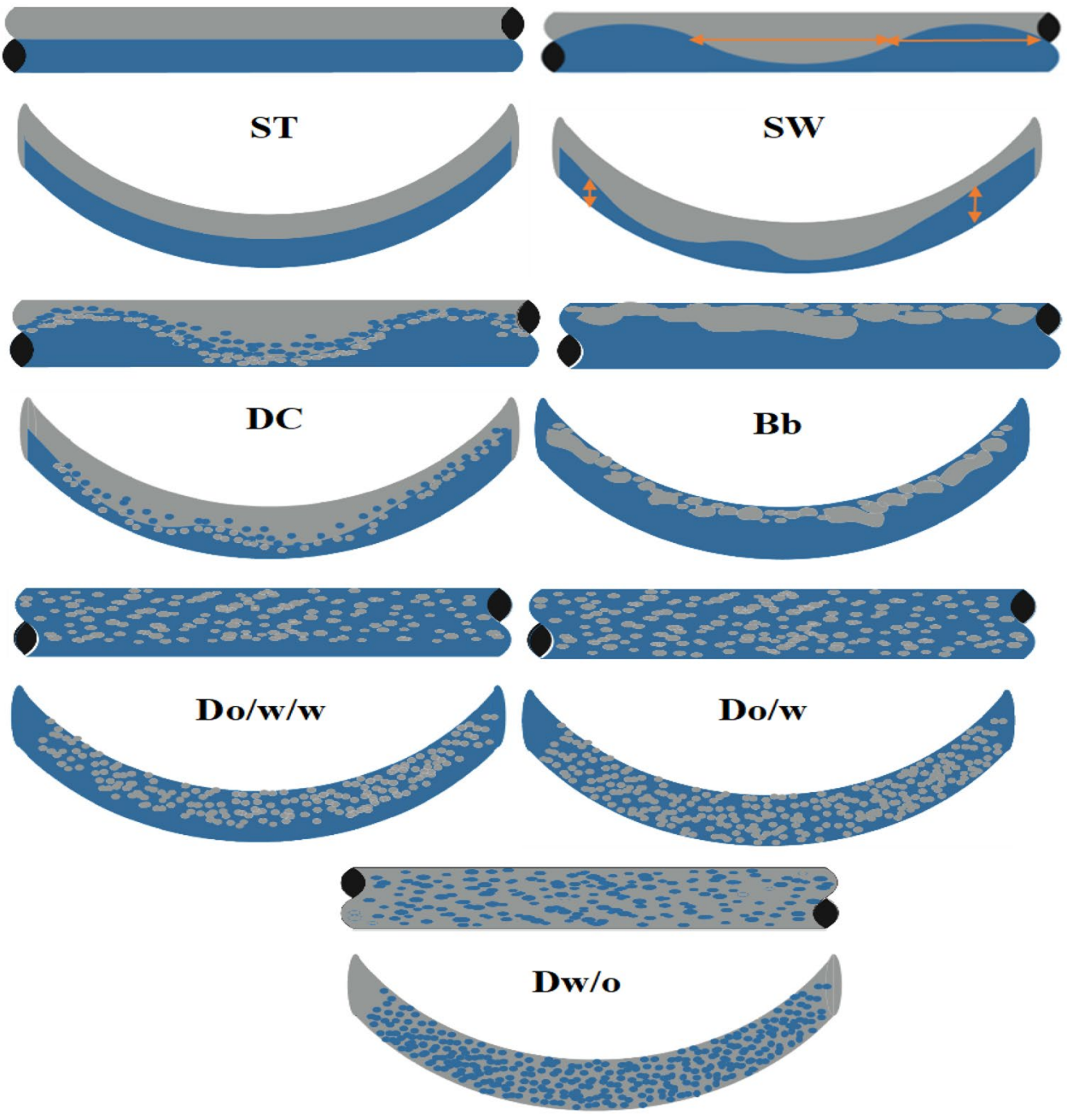

generated at the bend (or other fitting). Although the contribution of form drag is expected to decrease with downstream distance from the bend, there are insufficient data from which a conclusion can be drawn. Therefore, pressure drop measurements were carried out at two underdeveloped flow sections downstream of the bend with the view to characterise the pressure gradient associated with such flow.

In this work, a description of oil-water flow pattern/ phase distribution in and around $U$-bend is presented. The identified flow patterns are illustrated schematically in Fig. 1. Results of measured pressure gradient for oil-water flow at various locations in and around the $U$-bend are also presented. The results highlight the effect of the bend at various sections of the flow along with its cumulative effect on the combined region of flow affected by the bend.
Table 1 Test fluid properties at $22^{\circ} \mathrm{C}$

\begin{tabular}{lllll}
\hline Test fluids & $\begin{array}{l}\text { Density }(\mathrm{kg} / \\
\left.\mathrm{m}^{3}\right)\end{array}$ & $\begin{array}{l}\text { Viscosity } \\
(\mathrm{mPa} . \mathrm{s})\end{array}$ & $\begin{array}{l}\text { Surface } \\
\text { tension } \\
(\mathrm{mN} / \mathrm{m})\end{array}$ & $\begin{array}{l}\text { Oil-water } \\
\text { interfacial } \\
\text { tension } \\
(\mathrm{mN} / \mathrm{m})\end{array}$ \\
\hline $\begin{array}{l}\text { Tap water } \\
\begin{array}{l}\text { White min- } \\
\text { eral oil }\end{array}\end{array}$ & 898.2 & 1.0016 & 72.5 & 40.94 \\
\hline
\end{tabular}

\section{Materials and method}

\subsection{Materials}

See Table 1.

\subsection{Experimental flow loop and procedures}

The fluid properties measured are density, viscosity and surface tension. Density determination was done using 


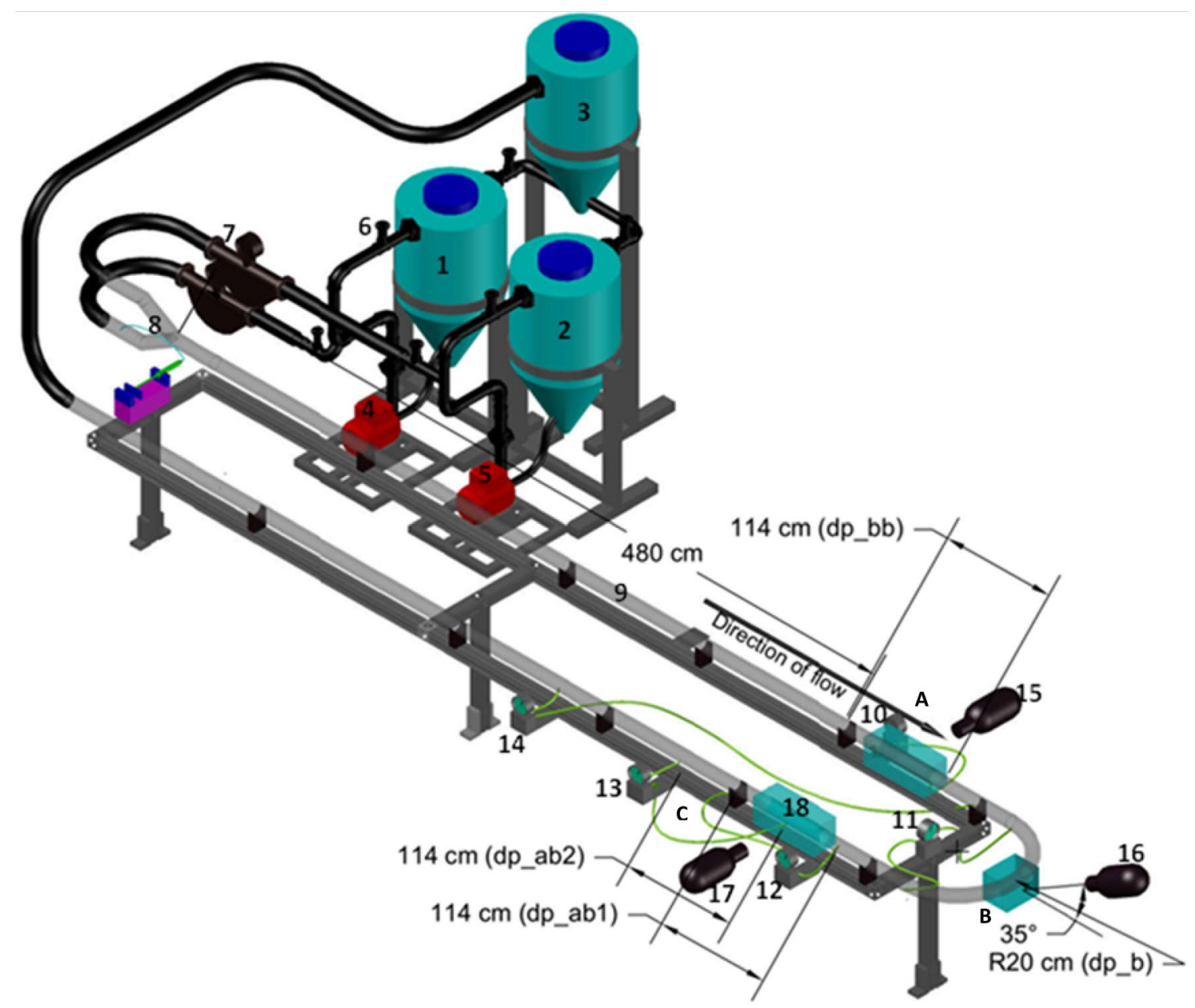

Fig. 2 Sketch of the experimental flow rig, 1. Water storage tank 2. Oil storage tank 3. Separation tank 4. Water CF pump 5. Oil CF pump 6. Gate valves 7. Coriolis mass flow meters 8. Y-junction 9. Clear PVC test Sect. 10. DP transducer (before bend) 11. DP trans-

the Coriolis flow meters. The Coriolis flow meter measured real-time densities of fluid flowing through it and record both the density and the corresponding temperatures. For these measurements, Coriolis flow meter model CMFS050M was used. The viscosity was measured using Fann Model 35A Couette rotational viscometer with accuracy $2.5 \% \mathrm{FS}$. Determination of surface tensions of oil and water as well as oil-water interfacial tension was done at Institut de Mécanique des Fluides de Toulouse using a Drop shape analyser (KRUSS model FM 40 Easydrop). Table 1 gives a summary of the measured fluid properties.

The experimental rig used for this study was a twophase flow loop assembled by the authors for oil-water flow experiments at the Richmond Field Station of University of California Berkeley (Fig. 2). The set-up included the holding, regulating and test sections. The holding section consisted of three conical-bottom inductor tanks each of 60-gallon capacity. The tanks were for oil, water and ducer (across bend) 12. DP transducer (after bend 1) 13. DP transducer (after bend 2) 14. DP transducer (bend + redeveloping flow section) 15. Camera (before bend) 16. Camera (bend) 17. Camera (after bend) 18. Rectangular view boxes

oil-water separation. The regulating section had two 1-hp centrifugal $C F$ pumps, connecting pipes, valves and two Coriolis mass flow meters (CMFS050M, 0-70 lbmin ${ }^{-1}$, accuracy: $\pm 0.05 \%$ ). Two flow meters were used for measuring the flowrates of oil and water. They also provided values for density, temperature among other fluid properties. The test section was made up of two parallel 19-mm ID clear $P V C$ pipes joined by a $U$-bend with radius of $100 \mathrm{~mm}$. The test section, divided into four parts, had five differential pressure transducers (four Yokogawa $0-35 \mathrm{mmHg}$ differential pressure (DP) transducers (model EJX 110A) and one Foxboro 0-850 mmHg DP transducer (model IDP10). One Yokogawa DP transducers was connected across a pair of pressure ports situated at 63.5 and $177.8 \mathrm{~cm}(114.3 \mathrm{~cm}$ apart) before the $U$-bend and used for measuring differential pressure of fully developed flow before the bend. A flow development length of $4.8 \mathrm{~m}$ was provided before the high-pressure port of this transducer. Another Yokogawa

\section{SN Applied Sciences}


DP transducer was connected across the $U$-bend on a pair of pressure ports that were $31.1 \mathrm{~cm}$ apart. A third Yokogawa DP transducer was connected across a pair of pressure ports situated 14.0 and $128.3 \mathrm{~cm}(114.3 \mathrm{~cm}$ apart $)$ downstream of the bend and used for differential pressure measurement immediately after the bend (i.e. redeveloping station 1). The last Yokogawa DP transducer was connected across a pair of pressure ports located at 63.5 and $177.8 \mathrm{~cm}(114.3 \mathrm{~cm}$ apart) downstream of the bend and used for taking measurement of pressure drop at a second section (i.e. station 2) further downstream of the bend (redeveloping Sect. 2). The Foxboro DP transducer was connected across a pair of pressure ports situated at $12.7 \mathrm{~cm}$ before the bend and $434.3 \mathrm{~cm}$ after the bend and used for taking measurement of the combined pressure drop due to bend and redeveloping section. This transducer served the purpose of validating results of the other transducers. All pressure ports were situated along the base of the test pipe, and the DP transducers were calibrated before use. Finally, each differential pressure or pressure drop measured was divided by the distance between the ports over which it was measured to obtain pressure gradient.

Three Basler Ace USB high-speed cameras were situated at $30.5 \mathrm{~cm}$ before-mid-way across-and $30.5 \mathrm{~cm}$ after-the bend for flow pattern identification. The cameras before and after the bend were installed at $0^{\circ}$ to the horizontal plane and perpendicular to the flow. The camera at the bend was installed at $35^{\circ}$ to the horizontal and perpendicular to the flow. The choice of angle was informed by the changes in the angle of the interface with changes in the superficial velocities of the phases. At lower flowrates when buoyancy effect dominated, the interface was almost horizontal (with water occupying the bottom section and oil the top). However, as relative velocity increased and centrifugal forces became significant, the interface inclined at an angle to the vertical and even became almost vertical at high relative velocities. In this case, water occupied the outer curve while oil occupied the inner side. All three flow-pattern-identifying locations were provided with rectangular viewing boxes filled with pure glycerine to minimise optical distortion due to pipe curvatures. Simultaneous image capturing was carried out with all three cameras at a frame rate of 57 $\mathrm{fps}$, and the exposure time was varied to suit the mixture flowrates. Flow pattern identification was carried out by careful examination of over 1000 close-up images combined with experimental notes and continuity of phases. All five differential transducers and the two Coriolis flow meters were connected to a computer for data acquisition via LabVIEW interface. For voltage measurements, 500 ohms $( \pm 5 \%)$ standard resistors were connected across the data acquisition terminals. However, to establish a base line and for reliability, direct voltage measurements were carried out across the terminals. The high-speed cameras were connected to three USB-3.0 ports on the computer.

Test measurements were carried out by varying the oil flowrate for a given water flowrate. The range of flowrates for water and oil was $0.13-1.1 \mathrm{~m} / \mathrm{s}$ and $0.04-0.95 \mathrm{~m} / \mathrm{s}$, respectively. The LabVIEW VI was set up to take simultaneous measurements from all the DP transducers, the two Coriolis flow meters and all three cameras. A minimum of 300 pressure and superficial velocity data sets were collected for each measurement run, and the runs were repeated at least three times at every measurement condition under consideration.

Uncertainties in superficial velocities $(\delta u)$ is a function of measurement uncertainty of mass flowrate $(\delta \dot{m})$, uncertainty of pipe inner diameter $(\delta d)$ and uncertainty of fluid density $(\delta \rho)$.

$u=\frac{4 \dot{m}}{\rho \pi d^{2}}$

$\delta u(\dot{m}, d, \rho)=\sqrt{\left(\frac{4}{\rho \pi d^{2}} \delta \dot{m}\right)^{2}+\left(\frac{-8 \dot{m}}{\rho \pi d^{3}} \delta d\right)^{2}+\left(\frac{-4 \dot{m}}{\rho^{2} \pi d^{2}} \delta \rho\right)^{2}}$

The uncertainty in tube diameter, mass flowrate and density are;

$\delta d= \pm 0.1 \mathrm{~mm}$

$\delta \dot{m}= \pm 0.05 \%$

$\delta \rho= \pm 0.05 \%$

The uncertainty in superficial water velocity at $u_{\mathrm{SW}}=0.13 \mathrm{~m} / \mathrm{s} \quad(\dot{m}=1.94 \mathrm{~kg} / \mathrm{s}) \quad$ and $u_{\mathrm{SW}}=1.10 \mathrm{~m} / \mathrm{s}$ $(\dot{m}=16.4 \mathrm{~kg} / \mathrm{s})$ are $(0.0363 \mathrm{~m} / \mathrm{s})$ and $(0.3074 \mathrm{~m} / \mathrm{s})$, respectively. That is;

$u_{\text {sW }}=0.13 \pm 0.0363 \mathrm{~m} / \mathrm{s}$

$u_{\mathrm{sW}}=1.10 \pm 0.3074 \mathrm{~m} / \mathrm{s}$

The uncertainty in pressure gradient $(\delta \Delta P)$ is mainly a function of the measurement accuracy of the differential pressure transducer, that is;

$\delta \Delta P \approx \Delta P_{\text {measured }} \pm 0.25 \%$

Uncertainties in pressure gradient may also be affected by the accuracy of horizontal alignment of the test section. Estimation of possible errors from this was not carried out and is expected to be negligible. Also, effort was made to ensure the test section was horizontal. Single-phase friction factor calculated from measured pressure drop was 
Fig. 3 Camera images of stratified flow. $\mathbf{a} U_{\text {so }}=0.12 \mathrm{~m} \mathrm{~s}^{-1}$, $U_{\mathrm{SW}}=0.13 \mathrm{~m} \mathrm{~s}^{-1}$.

b $U_{\text {SO }}=0.24 \mathrm{~m} \mathrm{~s}^{-1}$

$U_{\text {SW }}=0.13 \mathrm{~m} \mathrm{~s}^{-1}$. From left

to right: fully developed flow

before the bend, at the bend

and redeveloping flow after

the bend (a)

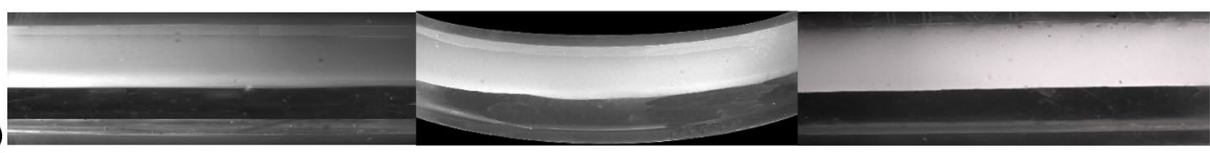

(b)

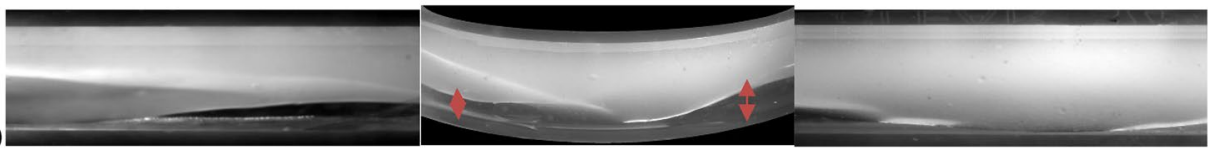

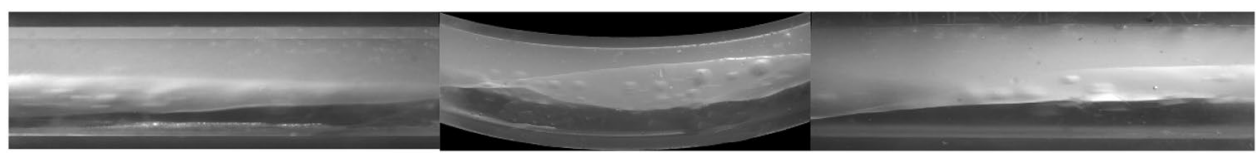

Fig. 4 Camera images of DC flow at $U_{S O}=0.1236 \mathrm{~m} \mathrm{~s}^{-1}$ and $U_{S W}=0.27 \mathrm{~m} \mathrm{~s}^{-1}$. From left to right: fully developed flow before the bend, at the bend and redeveloping flow after the bend

compared to the Blasius correlation (not shown) and the calculated friction factor where within $\pm 10 \%$ of the Blasius correlation.

\section{Results and discussion}

\subsection{Flow patterns in and around return bend}

Six flow regimes were identified over the measurement range and in all the test sections. These were stratified flow (ST), dual continuous or three-layer flow (DC), bubbly/plug flow $(\mathrm{Bb})$, dispersed oil in water and water layer (Do/w/w), dispersed oil in water (Do/w) and dispersed water in oil (Dw/o).

\subsubsection{Stratified flow (ST)}

At very low oil and water superficial velocities, buoyancy forces dominated in all test sections and the oil and water phases occupied the top and bottom sections of the pipe, respectively, separated by a smooth horizontal interface (Fig. 3a). With small increase in superficial velocity of either or both phases, long-wavelength asymmetric waves began to emerge at the interface. The crests of the waves were generally sharper than the troughs, which had longer wavelengths (Fig. 3b). As the effect of centrifugal forces became significant relative to buoyancy and inertia forces, the stratified flow characteristic at the bend showed a marked difference from those in the straight sections of the flow. The interfacial height at the entry of the bend was lower than that at the exit (Fig. 3b), and the interface was inclined to the vertical. The inclination was such that the interfacial height at the outer bend became higher than that at the inner bend. The angle of inclination of the interface increased with the increase in water superficial velocity at constant oil superficial velocity. Information on angle of inclination was only acquired for qualitative description and so this was estimated from few isolated images taken adjacent to the bend.

\subsubsection{Dual continuous (DC)/three-layer flow}

As the relative velocity of the phases was increased further, critical interfacial shear was reached and droplets began to form near the wavy interface (Fig. 4). Droplets of water were seen in the oil phase close to the interface and vice versa. The flow was still largely separated with clear oil and water phases at the top and bottom pipe sections, respectively. At higher mixture velocities, the wavy structure disappears and a layer of mixed oil and water bubbles form around the interface. This type of DC flow was described as threelayer flow by Al-Yaari et al. [24] (Fig. 5a). The DC or threelayer flow pattern characteristic in the straight sections was pretty similar. However, close inspection revealed that the bubble diameters for the redeveloping flow after the bend appeared smaller while the bubbles became more elongated. This was due to the shear forces impacted by the bend on the flow. Meanwhile, some unique features were identified at the bend. Firstly, the interface was inclined with the inclination as high as $75^{\circ}$ to the horizontal at higher mixture velocities just like in the case stratified flow. Secondly, the bubbles occupied a thin layer that was inclined to the horizontal, and thirdly, a thin intermittent oil film began to form at the outer wall whose thickness and duration scaled with oil superficial velocity (Fig. 5b).

\subsubsection{Bubbly/plug flow}

This occurred at moderate water flowrate and low oil superficial velocity. Elongated bubbles/plugs flowed at the top end of the pipe, whereas water formed the continuous phase.

\section{SN Applied Sciences}


Fig. 5 a Camera images of three-layer flow at $U_{\text {SO }}=0.48 \mathrm{~m} \mathrm{~s}^{-1}$ and $U_{S W}=0.27 m s^{-1}$. From left to right: fully developed flow before the bend, at the bend and redeveloping flow after the bend. $\mathbf{b}$ Camera image of tree-layer flow in U-bend with intermittent oil film at the outer wall
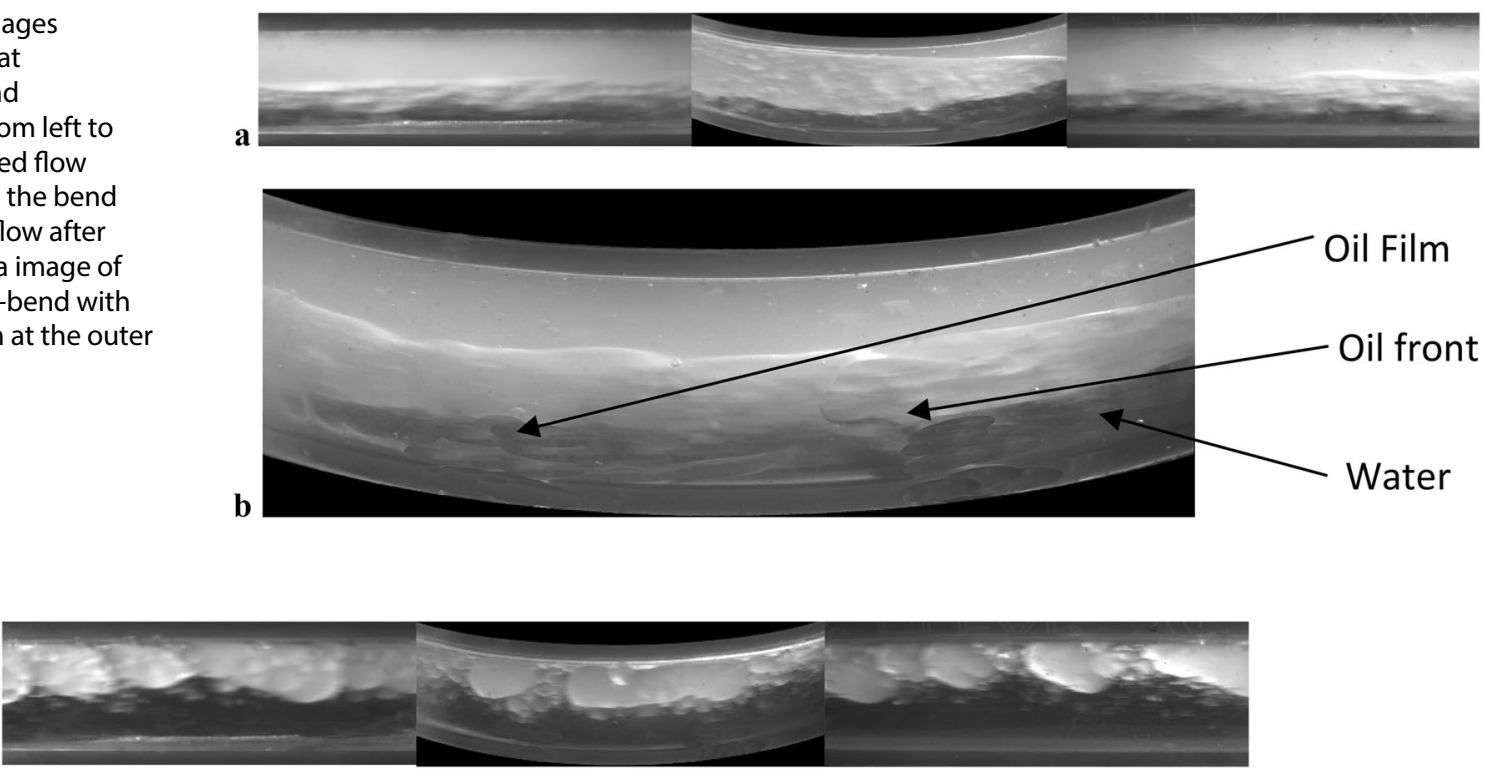

Fig. 6 Camera images of bubbly/plug flow at $U_{\mathrm{sO}}=0.12 \mathrm{~m} \mathrm{~s}^{-1}$ and $U_{\mathrm{SW}}=0.4 \mathrm{~m} \mathrm{~s}^{-1}$. From left to right: fully developed flow before the bend, at the bend and redeveloping flow after the bend

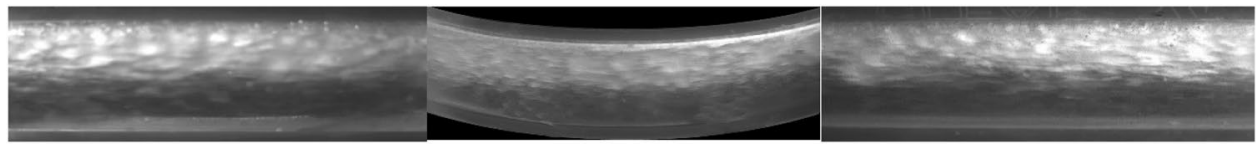

Fig. 7 Camera images of dispersed oil in water and water at $U_{\mathrm{SO}}=0.12 \mathrm{~m} \mathrm{~s}^{-1}$ and $U_{\mathrm{SW}}=0.54 \mathrm{~m} \mathrm{~s}^{-1}$. From left to right: fully developed flow before the bend, at the bend and redeveloping flow after the bend

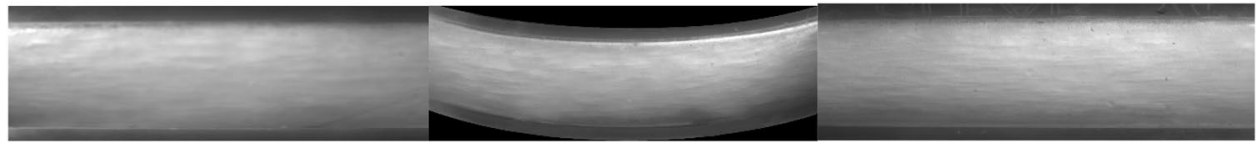

Fig. 8 Camera images of dispersed oil in water flow at $U_{\mathrm{SO}}=0.84 \mathrm{~m} \mathrm{~s}^{-1}$ and $U_{\mathrm{SW}}=1.07 \mathrm{~m} \mathrm{~s}^{-1}$. From left to right: fully developed flow before the bend, at the bend and redeveloping flow after the bend

Bubble diameter or plug length was similar to or longer than the pipe diameter. The bubbles/plugs were more closely packed in the hydrodynamically developed straight flow upstream of the bend and less so at the bend and after the bend. This was due to shear forces exacted by the bend on the bubbles (Fig. 6).

\subsubsection{Dispersed oil in water and water (Do/w/w)}

At high water superficial velocity and low oil superficial velocity, oil was dispersed in water. Do/w/w flow regime occurred in the range of $0.4 \leq U_{s W} \leq 1.07 \mathrm{~ms}^{-1}$, $U_{\text {so }} \leq 0.24 \mathrm{~ms}^{-1}$. In this range of superficial velocities, the buoyancy effect was still significant relative to inertia effect and a clear water layer flowed at the bottom section of the conduit. The thickness of this water layer decreased with the increase in oil fraction (Fig. 7).

\subsubsection{Dispersed oil in water (Do/w)}

At high mixture velocities in the water dominant regime, the oil phase was fully dispersed in the water phase (Fig. 8). In this flow regime, the inertia force was dominant over gravitational forces and the oil was dispersed all through the pipe cross section. It is important to state that with increasing oil superficial velocity, the transition from Do/w/w to Do/w did not always occur at the same oil superficial velocities in the hydrodynamically developed flow before bend and the redeveloping flow after the bend. The transition in the case of redeveloping flows 


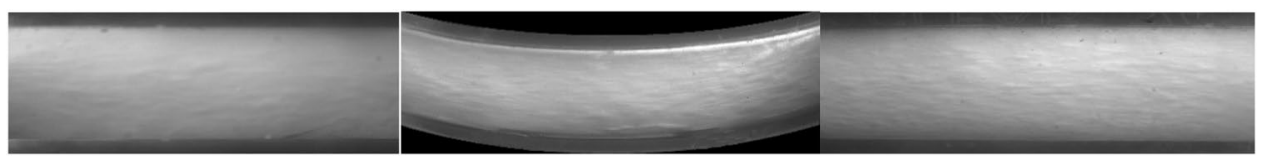

Fig. 9 Camera images of dispersed water in oil flow at $U_{\mathrm{SO}}=0.27 \mathrm{~m} \mathrm{~s}^{-1}$ and $U_{\mathrm{SW}}=0.96 \mathrm{~m} \mathrm{~s}^{-1}$. From left to right: fully developed flow before the bend, at the bend and redeveloping flow after the bend

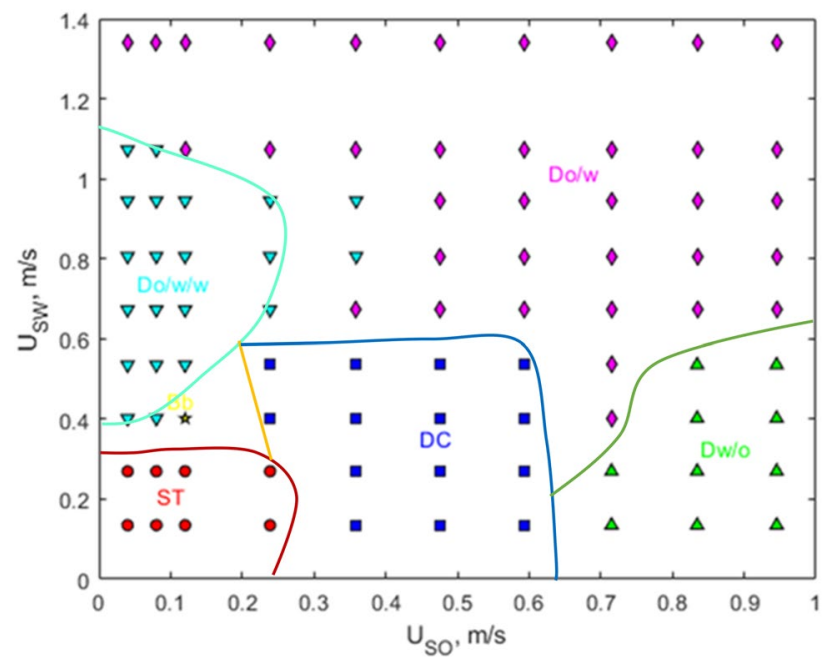

Fig. 10 Flow pattern maps for before the bend (solid lines) and at the bend (symbols)

sometimes occurred at higher superficial velocities of the oil phase. This can be attributed to the fact that the centrifugal forces at the bend, imposed phase separation on the mixture and this phase separation were carried on to the section after the bend, thereby creating a thin layer of clear water later at the bottom tube section. The difference was not always obvious due to limited image resolution. At the bend, Do/w flow pattern always seemed to have a very thin water layer at the outer wall of the bend, but oil droplets were intermittently suspended in this water layer.

\subsubsection{Dispersed water in oil (Dw/o)}

At high oil superficial velocities and low water superficial velocity, elliptical-shaped water droplets were dispersed throughout the oil continuous phase (Fig. 9).

\subsubsection{Flow pattern map}

Figure 10 represents the flow pattern map upstream of (i.e. before) the bend superimposed on the flow pattern map at the bend. It should be noted that the flow pattern maps at the bend and redeveloping downstream of (i.e. after) the bend are very similar. Hence, the reason why flow pattern map of the redeveloping flow after the bend was not presented. The obvious difference between the flow pattern maps before and at the bend (or redeveloping downstream of the bend) was that Do/w/w persisted over a wider range of oil superficial velocity for the later. In the flow pattern map of Sharma et al. [14], the flow patterns were categorised into stratified flow, plug flow and kerosine dispersed. Similar to Sharma et al. [14], stratified flow regime was reported at low water and low oil superficial velocities. However, at low water and high oil superficial velocities, dispersed water in oil (Dw/o) was observed in the current study as opposed to stratified flow pattern reported in the work of Sharma et al. [14]. This could be associated with the lower density oil used by Sharma et al. [14] $\left(\rho_{\mathrm{o}}=787 \mathrm{~kg} / \mathrm{m}^{3}\right)$ relative to that used in the current work $\left(\rho_{\mathrm{o}}=835 \mathrm{~kg} / \mathrm{m}^{3}\right)$. The higher density difference between water and oil in the work investigation of Sharma et al. [14] promotes phase separation due to buoyancy, thereby increasing the region of stratified flow to higher mixture velocities. The regime corresponding to plug flow in the flow pattern map of Sharma et al. [14] mostly corresponds to $\mathrm{DC} / \mathrm{Bb}$ in the current study. Similarly, the dispersed kerosine in water regime reported by Sharma et al. [14] mostly corresponds to Do/w and Do/w/w regimes in the current study.

\subsection{Pressure gradient for co-current oil and water flow in and around $\mathrm{U}$-bend}

$\ln [1,23,25]$.

In specific terms, plots of pressure gradient of co-current oil and water flow before the bend, at the bend and at two redeveloping locations downstream of the $U$-bend versus oil fraction $\left(U_{\mathrm{SO}} /\left(U_{\mathrm{SW}}+U_{\mathrm{SO}}\right)\right)$ at various water superficial velocities, are presented. The general characteristics of the curves are similar in all test locations, but they reveal an increase in pressure gradient with oil fraction. The later trend agrees with previous study on co-current oil and water flow in horizontal tubes $[1,23,25]$.

In specific terms, $[1,23,25]$.

In specific terms, a shows the pressure gradient versus oil fraction for oil-water flows in the fully developed flow section upstream of the bend. Over the measurement conditions considered in the present study, there were two distinct regions in the plots corresponding to separated flow and mixed flow regimes. Between these two flow 

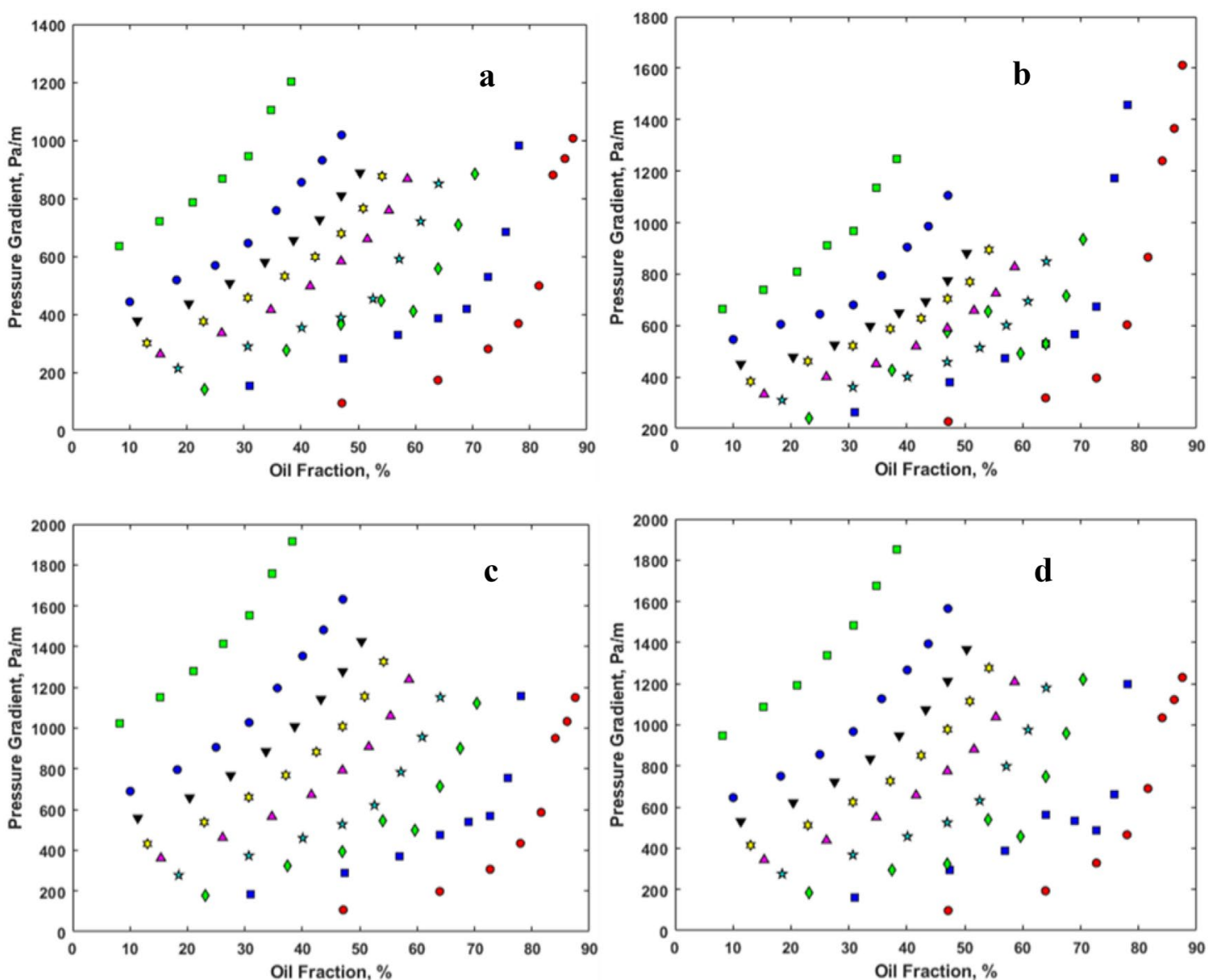

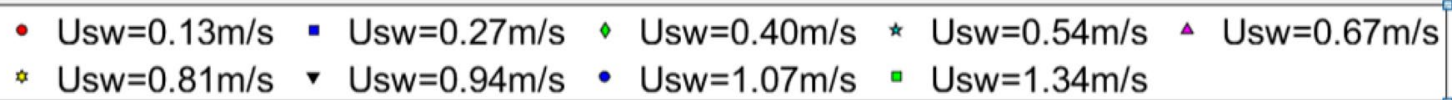

Fig. 11 Pressure gradient variation with oil fraction at different water superficial velocities. a Before the bend, $\mathbf{b}$ at the bend, $\mathbf{c}$ location 1 after the bend, $\mathbf{d}$ location 2 after the bend

regimes, there appeared to be a transition region (dash curves in $[1,23,25]$.

In specific terms, (a) was always found within the range of $0.4 \leq U_{\mathrm{sw}} \leq 0.54 \mathrm{~m} / \mathrm{s}$. As oil and water velocities were increased, the flow pattern moved from separated flow to mixed flow. This behaviour was also observed at the bend and downstream of the bend $[1,23,25]$.

In specific terms, (b-d) albeit to different degrees. For example, the transition in $[1,23,25]$.

In specific terms, c, as indicated by circled points, corresponds to a transition from dual continuous flow (separated flow) to dispersed flow (mixed flow) downstream of the bend. In the DC flow, the mixing was restricted to a region close to the interface and droplets of either phases were observed in the other phase $[1,26]$. At transition, as in this case, there was significant mixing in the entire oil layer and water bubbles were observed close to the top section of the pipe. It was also observed that the continuous oil layer at the top-half of the pipe began to break up resulting in an oscillating flow behaviour. This resulted in a significant decrease in time averaged pressure gradient because the contribution of the more viscous phase (oil) to wall frictional losses was reduced (Fig. 11).

To compare the pressure gradient at the various test locations in and around the $U$-bend, plots of pressure gradient versus oil fraction at selected water superficial velocities are provided in Fig. 12. At low water superficial velocity of $0.13 \mathrm{~m} / \mathrm{s}$ (Fig. 12a), pressure gradient curves before and after the bend collapsed into a single curve especially at low oil fraction the pressure while that at the bend was noticeably higher than those upstream and downstream of the bend at lower oil fractions. This is because at low mixture velocities, the contribution of secondary flows to pressure drops relative to those of wall and interfacial frictions is significant. In addition, pressure gradient curves for all the test locations at this flow condition 

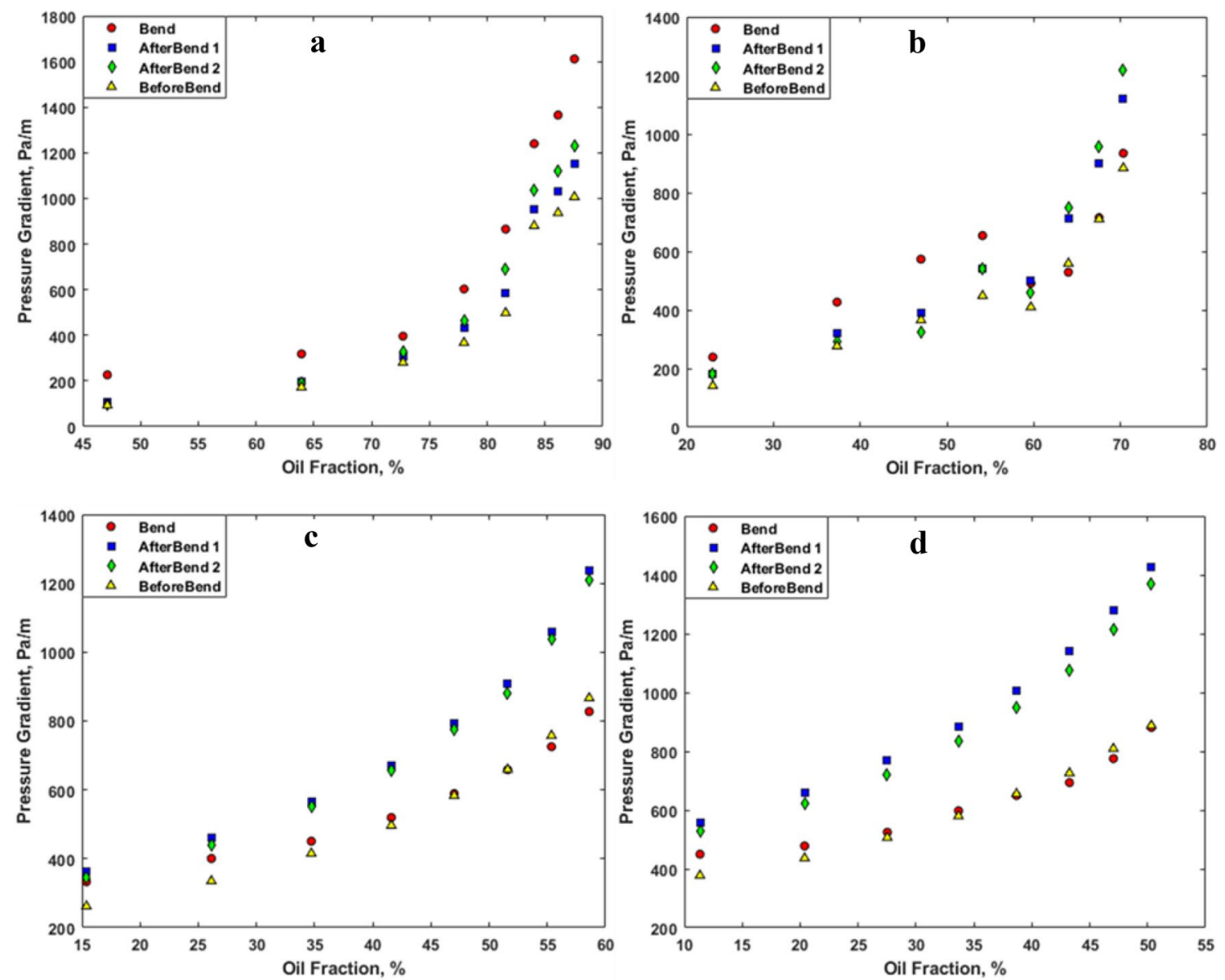

Fig. 12 Comparison of pressure gradients in and around $U$-bend for oil-water flows at selected water superficial velocities. a $U_{\mathrm{sW}}=0.13 \mathrm{~m} / \mathrm{s}, \mathbf{b} U_{\mathrm{sW}}=0.40 \mathrm{~m} / \mathrm{s}, \mathbf{c} U_{\mathrm{sW}}=0.67 \mathrm{~m} / \mathrm{s}, \mathbf{d} U_{\mathrm{sW}}=0.94 \mathrm{~m} / \mathrm{s}$

also showed a moderate increase at low oil fractions and an exponential increase with high oil fractions. This was due to the increase in the mixture viscosity as the oil fraction increased. With the increase in the flowrate of water, the pressure drops showed different trends in all test locations and at various oil fractions (Fig. 12b). At lower oil fraction (and lower mixture velocity), pressure gradient at the bend was expectedly higher than those before and after the bend. At higher mixture velocity, where turbulence dominated, the pressure gradients at the bend and those before the bend converged. Interestingly, at higher mixture velocity/oil fraction, the pressure gradients for the two redeveloping locations after the bend were higher than those before the bend and at the bend. This is because flow disturbances after the bend became significant and the contribution of form drag to pressure losses was quite significant at these high mixture velocities. The difference in pressure gradient among the sections was not obvious from flow visualisation due to the limited resolution of the cameras. At even higher mixture velocities (Fig. 12c, d), this trend of the pressure gradients became even much more pronounced. At such high Reynolds number (Fig. 12d), pressure drop after the bend corresponded to over $60 \%$ of the total pressure drop in the combined test section (measurement from Foxboro DP cell for combined section). The difference between these two figures is that the pressure gradients at the bend were higher than those before the bend at low oil fractions, but they both converged at high oil fractions in Fig. 12c. This is attributed to the wall and interfacial effect that dominated in Fig. 12d, and therefore, both pressure gradient curves showed similar characteristics irrespective of oil fraction.

Results of pressure gradients versus water superficial velocities are also presented to highlight the effect of changes in $U_{S W}$ on pressure gradient in and around bends (Fig. 13). At low $U_{S W}$, the pressure gradient in the bend superseded those before the bend due to the significant contribution of secondary flows to pressure drops relative to turbulence (Fig. 13a). At higher water superficial velocities, a larger percentage of pressure drops occurred at the redeveloping sections after the bend. In addition, the pressure gradients immediately after the bend were 

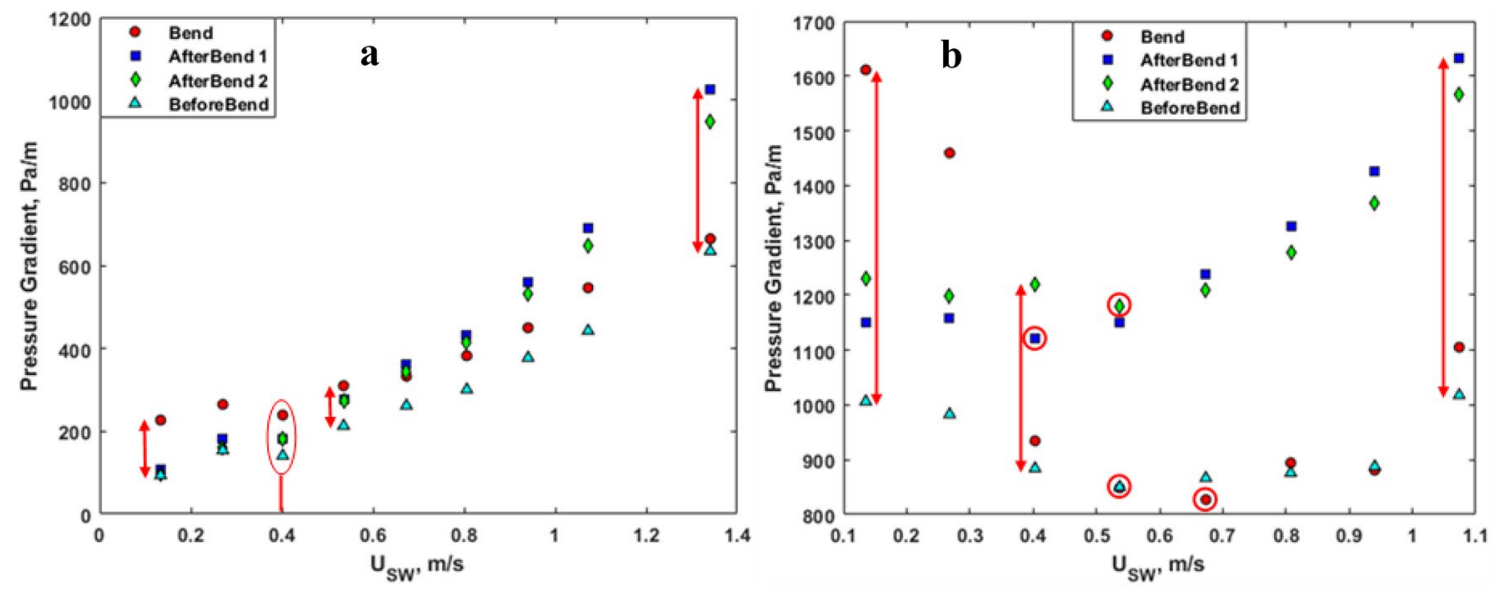

Fig. 13 Comparison of the pressure gradients in and around U-bends for oil-water flows at selected $U_{\mathrm{sO}} \cdot \mathbf{a} U_{\mathrm{sO}}=0.12 \mathrm{~m} / \mathrm{s}, \mathbf{b} U_{\mathrm{sO}}=0.96 \mathrm{~m} / \mathrm{s}$

slightly higher than those further downstream of the bend. This suggests that the contribution of form drag to pressure drops is a function of the level of flow redevelopment. Interestingly, the trajectory of the curves for both redeveloping locations after the bend is similar and this suggests that similar forces were at play in both redeveloping locations. Figure 13a also highlights the region of flow transition $\left(0.4 \leq U_{\mathrm{sw}} \leq 0.54 \mathrm{~m} / \mathrm{s}\right)$ discussed earlier. In the water dominant regime, increase in $U_{S W}$ resulted in increase in the difference between the pressure gradients in the redeveloping flows and those before and at the bend. At significantly high oil superficial velocity (Fig. 13b), the characteristics of the curves are somewhat different and the transition velocities depended on the test location. For instance, at $U_{s w}<0.54 \mathrm{~m} / \mathrm{s}$ where the oil phase dominated, the pressure gradients generally decreased with the increase in $U_{\mathrm{SW}}$, and water acted as a drag reducing agent. As the mixture velocity was increased further, minimum pressure gradients were attained after which further increase in flowrate resulted in higher pressure gradients. At these high mixture velocities, the mixture Reynolds numbers, which was responsible for the turbulence intensity, largely determined the pressure gradients.

Overall, the flow patterns identified in the current work were consistent with that of Sharma et al. [14]. Similar to Sharma et al. [14], stratified flow regime was reported at low water and low oil superficial velocities. However, at low water and high oil superficial velocities, dispersed water in oil (Dw/o) was observed in the current study as opposed to stratified flow pattern reported in the work of Sharma et al. [14]. This could be associated with the lower density oil used by Sharma et al. [14] $\left(\rho_{\mathrm{o}}=787 \mathrm{~kg} / \mathrm{m}^{3}\right)$ relative to that used in the current work $\left(\rho_{\mathrm{o}}=835 \mathrm{~kg} / \mathrm{m}^{3}\right)$. The higher density difference between water and oil in the work investigation of Sharma et al. [14] promotes phase separation due to buoyancy, thereby increasing the region of stratified flow to higher mixture velocities. The regime corresponding to plug flow in the flow pattern map of Sharma et al. [14] mostly corresponds to $\mathrm{DC} / \mathrm{Bb}$ in the current study. However, in the current work, some interesting phase distribution behaviour was observed within the bend such as the one involving intermittent oil film at the outer wall of the bend. The dispersed kerosine in water regime reported by Sharma et al. [14] mostly corresponds to Do/w and Do/w/w regimes in the current study. However, in the current work some differences in phase distribution after the bend (underdeveloped flow section) were observed (relative to developed flow before the bend in both current work and the work of Sharma et al. [14]). Also, pressure gradient results were generally consistent with reports of Yusuf et al. [21], and Al-Wahaibi et al. [23] (oil-water flow in straight pipe) and Sharma et al. $[13,14]$ (oil-water flow in U-bend) within comparable oil superficial velocity range. Similar trends were also reported by [27] in their gas-liquid two-phase experiments in U-bends. The approach adopted in the current work which involved the determination of pressure gradient at various locations of flow redevelopment as well as across the bend over a wide range of flowrate provides a more comprehensive characterisation of pressure drop distribution in and around $U$-bends.

\section{Conclusion}

Experimental measurements of the flow patterns and pressure drops for oil-water flow in and around U-bend were carried out with the view of investigating the effect of the bend on oil-water flow characteristics. Based on results obtained, the following conclusion could be drawn. 
- Flow patterns in the various test locations were largely similar. However, the transition mixture velocity of the flow before the bend sometimes different from those at the bend and after the bend.

- Pressure gradients at all the test locations increased with both oil fraction and water superficial velocity, and there was a change of pressure gradient profile at the point of inversion from oil dominant to water dominant flow regime.

- Pressure gradients differed with respect to test location, and the difference was strongly related to the superficial velocity of the phases and the flow pattern. In general, at high mixture velocity, pressure gradients at the redeveloping flow after the bend were higher than those before the bend and those at the bend. At low mixture velocity, pressure gradients at the bend were higher than those before and those after the bend.

- Pressure gradients immediately after the bend were generally higher than those further downstream after the bend. This indicates that there was decrease in pressure drops with level of flow development downstream of the bend.

Acknowledgements The authors wish to thank the Flow Group of University of California Berkeley and in particular Simo A. Mäkiharju, Eric Thacher and Daniel Grieb for the support provided for this research. The authors also wish to acknowledge the free donation of HPAM polymer for this research by BASF-Chemicals USA.

Funding This research work was funded by United States J.W. Fulbright Program/U.S. Department of State and Institute of International Education (IIE).

Availability of data and material Raw and processed experimental are properties of the authors and may be made available were requested. The data reported in this work showed no discrepancies. Materials and experimental facility used for this work is at Richmond Field Station of the University of California Berkeley, USA. The version of this manuscript that has not been peer-reviewed is available on preprints.org as: https://doi.org/10.20944/Preprints202005.0278.V1.

\section{Compliance with ethical standards}

Conflict of interest The authors wish to declare that there are no conflicts of interest with the publication of this manuscript.

Open Access This article is licensed under a Creative Commons Attribution 4.0 International License, which permits use, sharing, adaptation, distribution and reproduction in any medium or format, as long as you give appropriate credit to the original author(s) and the source, provide a link to the Creative Commons licence, and indicate if changes were made. The images or other third party material in this article are included in the article's Creative Commons licence, unless indicated otherwise in a credit line to the material. If material is not included in the article's Creative Commons licence and your intended use is not permitted by statutory regulation or exceeds the permitted use, you will need to obtain permission directly from the copyright holder. To view a copy of this licence, visit http://creativeco mmons.org/licenses/by/4.0/.

\section{References}

1. Edomwonyi-Otu LC, Angeli P (2015) Pressure drop and holdup predictions in horizontal oil-water flows for curved and wavy interfaces. Chem Eng Res Des 93:55-65

2. Mandal MM, Aggarwal P, Nigam KDP (2011) Liquid-liquid mixing in coiled flow inverter. Ind Eng Chem Res 50(23):13230-13235

3. Ayegba PO, Abdulkadir M, Hernandez-Perez V, Lowndes IS, Azzopardi BJ (2017) Applications of artificial neural network (ANN) method for performance prediction of the effect of a vertical $90^{\circ}$ bend on an air-silicone oil flow. J Taiwan Inst Chem Eng 10:1-6

4. Ayegba PO, Abdulkadir M (2016) Prediction of average void fraction and pdf of void fraction in vertical 900 bend for air-silicone oil flow using multilayer perceptron (Mlp) codes. Int J Lean Think 7(2):80-105

5. Ghosh S, Mandal TK, Das G, Das PK (2009) Review of oil water core annular flow. Renew Sustain Energy Rev 13(8):1957-1965

6. Strazza D, Poesio $P(2012$ ) Experimental study on the restart of core-annular flow. Chem Eng Res Des 90(11):1711-1718

7. Tripathi S, Bhattacharya A, Singh R, Tabor RF (2015) Lubricated transport of highly viscous non-Newtonian fluid as core-annular flow: a CFD study. Procedia IUTAM 15:278-285

8. Loh WL, Premanadhan VK (2016) Experimental investigation of viscous oil-water flows in pipeline. J Pet Sci Eng 147:87-97

9. Ameri M, Tirandaz N (2017) Two phase flow in a wavy coreannular configuration through a vertical pipe: analytical model for pressure drop in upward flow. Int J Mech Sci 126(February):151-160

10. Goldstein A, Ullmann A, Brauner N (2017) Exact solutions of core-annular laminar inclined flows. Int J Multiph Flow 93:178-204

11. van Duin E, Henkes R, Ooms G (2018) "Influence of oil viscosity on oil-water core-annular flow through a horizontal pipe," Petroleum, pp 1-7

12. Pietrzak M (2014) Flow patterns and volume fractions of phases during liquid - liquid two-phase flow in pipe bends. Exp Therm Fluid Sci 54:247-258

13. Sharma M, Ravi P, Ghosh S, Das G, Das PK (2011) Hydrodynamics of lube oil - water flow through $180^{\circ}$ return bends. Chem Eng Sci 66(20):4468-4476

14. Sharma M, Ravi P, Ghosh S, Das G, Das PK (2011) Studies on low viscous oil-water flow through return bends. Exp Therm Fluid Sci 35(3):455-469

15. Angeli P, Hewitt GF (2000) Flow structure in horizontal oil-water flow. Int J Multiph Flow 26(7):1117-1140

16. Ghosh S, Das G, Kumar P (2011) Simulation of core annular in return bends-a comprehensive CFD study. Chem Eng Res Des 89(11):2244-2253

17. Zhou Y, Shah SN, Gujar PV (2004) Effects of coiled-tubing curvature on drag reduction of polymeric fluids. SPE Prod Oper 21(1):134-141 
18. Gasljevic K, Matthys EF (2009) Friction and heat transfer in dragreducing surfactant solution flow through curved pipes and elbows. Eur J Mech / B Fluids 28(5):641-650

19. Ayegba PO, Edomwonyi-Otu LC, Yusuf N, Abubakar A (2019) Results in Materials Experimental and neural network modelling of polymer drag reduction in 180 bends. Results Mater 1:1-11

20. Ayegba PO, Edomwonyi-Otu LC (2020) Turbulence statistics and flow structure in fluid flow using PIV technique: a review. Eng Rep 2(3):e12138

21. Yusuf N, Al-Wahaibi Y, Al-Wahaibi T, Al-Ajmi A, Olawale AS, Mohammed IA (2012) Effect of oil viscosity on the flow structure and pressure gradient in horizontal oil-water flow. Chem Eng Res Des 90(8):1019-1030

22. Edomwonyi-Otu LC, Angeli P (2019) Separated oil-water flows with drag reducing polymers. Exp Therm Fluid Sci 102:467-478

23. Al-Wahaibi T, Al-Wahaibi Y, Al-Ajmi A, Al-Hajri R, Yusuf N, Olawale AS (2014) Experimental investigation on flow patterns and pressure gradient through two pipe diameters in horizontal oilwater flows. J Pet Sci Eng 122:266-273
24. Al-Yaari M, Al-Sarkhi A, AbU-Sharkh B (2012) Effect of drag reducing polymers on water holdup in an oil-water horizontal flow. Int J Multiph Flow 44:29-33

25. Edomwonyi-Otu LC, Chinaud M, Angeli P (2015) Effect of drag reducing polymer on horizontal liquid-liquid flows. Exp Therm Fluid Sci 64:164-174

26. Edomwonyi-Otu LC, Barral AH, Angeli P (2014) Oil-Water flow characteristics in pipes of different diameters. Niger J Mater Sci Eng 5(1):35-40

27. Mandal SN, Das SK (2001) Pressure losses in bends during two-phase gas - Newtonian liquid flow. Ind Eng Chem Res 40(10):2340-2351

Publisher's Note Springer Nature remains neutral with regard to jurisdictional claims in published maps and institutional affiliations. 